\title{
Downregulation of CASR expression and global loss of parafibromin staining are strong negative determinants of prognosis in parathyroid carcinoma
}

Janneke E Witteveen ${ }^{1}$, Neveen AT Hamdy ${ }^{1}$, Olaf M Dekkers ${ }^{1,2}$, Job Kievit ${ }^{3}$, Tom van Wezel $^{4}$, Bin T Teh ${ }^{5}$, Johannes A Romijn ${ }^{1}$ and Hans Morreau ${ }^{4}$

${ }^{1}$ Department of Endocrinology and Metabolic Diseases, Leiden University Medical Center, Leiden, The Netherlands; ${ }^{2}$ Department of Clinical Epidemiology, Leiden University Medical Center, Leiden, The Netherlands; ${ }^{3}$ Department of Surgery, Leiden University Medical Center, Leiden, The Netherlands; ${ }^{4}$ Department of Pathology, Leiden University Medical Center, Leiden, The Netherlands and ${ }^{5}$ Laboratory of Cancer Genetics, Van Andel Research Institute, Grand Rapids, MI, USA

\begin{abstract}
Parathyroid carcinoma is associated with mutations in the HRPT2/CDC73 gene and with decreased parafibromin and calcium-sensing receptor (CASR) expression, but in some cases establishing an unequivocal diagnosis remains a challenge. The aim of our study was to evaluate the prognostic value of CASR and parafibromin expression and of HRPT2/CDC73 mutations in patients with an established diagnosis of parathyroid carcinoma. Data on survival and disease-free survival were obtained from hospital records of 23 patients with an established diagnosis of parathyroid carcinoma in whom CASR and parafibromin expression and HRPT2/CDC73 mutation analyses were available from paraffin-embedded pathological specimens. KaplanMeier curves were used for survival analysis. Downregulation of CASR expression, global loss of parafibromin staining and a HRPT2/CDC73 mutation were, respectively, found in $7(30 \%), 13(59 \%)$ and $4(17 \%)$ patients, and were associated with, respectively, 16-fold, 4-fold and 7-fold increased risk of developing local or distant metastasis. These findings suggest that although downregulation of CASR expression, global loss of parafibromin staining and mutations in the HRPT2/CDC73 gene are tools of proven value to assist in establishing a diagnosis of parathyroid carcinoma, their absence does not exclude it. Notwithstanding, we demonstrate a significant added value of these markers as strong determinants of increased malignant potential and thus as negative prognostic markers in this malignancy.

Modern Pathology (2011) 24, 688-697; doi:10.1038/modpathol.2010.236; published online 14 January 2011
\end{abstract}

Keywords: CASR; disease-free survival; HRPT2/CDC73 mutation; overall survival; parafibromin; parathyroid carcinoma; $M E N-1$ mutation

Parathyroid carcinoma is a rare disorder, accounting for $0.1-5.0 \%$ of all cases of primary hyperparathyroidism. ${ }^{1-6}$ The disease presents at a mean age of 50 years and, contrary to sporadic hyperparathyroidism, is equally likely to occur in men and women. . $^{1,3-5,7}$ Clinical manifestations are those of severe primary

Correspondence: Dr $\mathrm{H}$ Morreau, MD, PhD, Department of Pathology, Leiden University Medical Center, Albinusdreef 2, 2333 ZA, Leiden, The Netherlands.

E-mail: J.Morreau@lumc.nl

Received 30 September 2010; revised 16 November 2010; accepted 16 November 2010; published online 14 January 2011 hyperparathyroidism, often associated with renal and skeletal complications..$^{1,3-5,8}$ A neck mass is palpable in $30-76 \%$ of patients. ${ }^{1,5,9,10}$ The diagnosis of parathyroid carcinoma is suspected by intraoperative features of local invasion and confirmed by the World Health Organization (WHO) histopathological criteria for parathyroid carcinoma. These include vascular invasion, perineural space invasion, capsular penetration with growth into adjacent tissues and/or metastasis. ${ }^{11}$ Despite the availability of these histopathological criteria, the diagnosis of parathyroid carcinoma remains a challenge in some cases, and markers such as mutations in 
the tumor-suppressor gene HRPT2/CDC73, ${ }^{12-15}$ and global loss of expression of its protein, parafibromin, ${ }^{16,17}$ are proven valuable tools frequently used to assist in establishing a diagnosis of this malignancy.

Mutations in the HRPT2/CDC73 gene, which encodes for the protein parafibromin, ${ }^{18}$ are associated with the hyperparathyroidism-jaw tumor syndrome (HPT-JT; OMIM 607393). Patients with HPT-JT syndrome have a 15-24\% chance of developing parathyroid carcinoma, and loss of heterozygosity of chromosome 1q, the location of the HRPT2/CDC73 gene, is reported to be found in $55 \%$ of parathyroid carcinomas. ${ }^{1,17,19-21}$

The calcium-sensing receptor (CASR) regulates not only the synthesis and secretion of parathyroid hormone, but also the proliferation of the parathyroid glands. We have previously reported that $31 \%$ of parathyroid carcinomas had irregular or absent staining for CASR compared with only $1 \%$ of sporadic adenomas. ${ }^{21}$ We have also demonstrated, using gene expression analysis, a significant downregulation of the CASR in patients with sporadic carcinoma or familial HPT-JT compared with patients with sporadic primary hyperparathyroidism, primary hyperparathyroidism due to $M E N-1$ or $M E N-2$ mutations or primary hyperparathyroidism due to lithium use. ${ }^{22}$ Downregulation of CASR has been previously shown to be a negative prognostic factor in colon carcinoma, ${ }^{23-25}$ but there are no available data on the prognostic value of downregulation of CASR in parathyroid carcinoma. There are also no available data on the prognostic value of loss of parafibromin staining in the presence of a HRPT2/CDC73 mutation.

The aim of our study was to evaluate the prognostic value of downregulation of CASR expression, loss of parafibromin staining and the presence of mutations in the HRPT2/CDC73 gene in patients with a WHO criteria-based diagnosis of parathyroid carcinoma.

\section{Materials and methods}

\section{Study Population}

We identified 29 patients who had initial surgery for parathyroid carcinoma in various hospitals in the Netherlands in the period 1985-2000. All resected tumors were screened for somatic and/or germline mutations in the HRPT2/CDC73 and the MEN-1 genes, and immunohistochemical staining for parafibromin and CASR was performed on all 29 pathological specimens in the Department of Pathology of the Leiden University Medical Center. ${ }^{21}$ Four patients were lost to follow-up, one patient died (normocalcemic) of a myocardial infarction 4 months after initial parathyroidectomy and one patient did not meet the histopathological WHO criteria for parathyroid carcinoma and were excluded from the final analysis. A total of 23 patients were thus included in the study. The study was approved by the local ethics committee of the Leiden University Medical Center.

\section{Methods}

Clinical data were obtained from the hospital records of the 23 patients included in the study, with special emphasis on time to development of local recurrence and/or distant metastasis and when applicable time and cause of death. Data on initial clinical presentation were available in 16 of the 23 patients $(70 \%)$ including physical examination of the neck in 14 of the 23 patients (61\%). Serum biochemistry before initial surgery was available in 20 of the 23 patients (87\%) and hypercalcemia was documented in all patients, although exact concentrations were not available in 3 patients. Operative and pathology data were available in all 23 patients.

\section{End points of the study}

The primary end point of the study was the development of local recurrence and/or distant metastasis. Recurrence was defined as recurrence of an increase in serum calcium and parathyroid hormone concentrations after a period of normalization of at least 6 months after initial surgery. Local recurrence was defined on the basis of a surgically removed lesion from a site adjacent to the primary tumor that was histologically confirmed to be parathyroid carcinoma. Distant metastasis was defined as radiologically and/or surgically and histologically proven distant metastasis diagnosed at least 6 months after successful excision of a primary tumor.

The secondary end point of the study was death related to parathyroid carcinoma, which was defined as death because of parathyroid hormonerelated uncontrollable hypercalcemia. Patients who died from other causes in the setting of normocalcemia were censored at the time of death.

Histopathological diagnosis of parathyroid carcinoma The histopathological diagnosis of parathyroid carcinoma was established using the WHO criteria for this malignancy. These include vascular invasion, perineural space invasion, capsular penetration with growth into adjacent tissues and/or metastasis. ${ }^{11}$ The presence of a trabecular pattern, fibrous bands and/or multiple mitoses were considered to be minor criteria. All pathological specimens were re-evaluated in our Department of Pathology using the WHO criteria and only those fulfilling these criteria were included in the analysis.

\section{Pathological analysis}

Pathological analysis of the primary tumor has been reported in detail in previous publications. ${ }^{14,21}$ In brief, formalin-fixed, paraffin-embedded (FFPE) 
specimens of the primary parathyroid carcinomas of all 23 patients were screened for HRPT2/CDC73 and $M E N-1$ gene mutations, ${ }^{14}$ and all were immunohistochemically stained for parafibromin and CASR (Figures 1 and 2). ${ }^{17,21}$ Downregulation of CASR expression was defined as irregular or absent staining pattern for CASR on the membrane of parathyroid tumor cells. Focal loss of parafibromin staining was previously defined as the absence of nuclear staining in variably sized regions. Global loss of parafibromin staining was defined as the absence of nuclear staining in all tumor tissue.
The three identified germline HRPT2/CDC73 mutations were positioned on exon 2 (c.176C $>\mathrm{T}$, pSer59Phe, patient no. 1), exon 7 (c.687_688delAG, p.Arg229fsX, patient no. 23) and exon 7 (c.691_692insT, p.Trp231fsX35, patient no. 21), respectively. ${ }^{14}$ The two identified somatic HRPT2/ CDC73 mutations were positioned on exon 1 (c128G > A, pTrp43fsX, patient no. 21) and exon 2 (c.165delC, pTyr55fsX, patient no. 6), respectively. ${ }^{14}$ The three identified somatic $M E N-1$ mutations were positioned on exon 2 (c.168_171delCAAC, pAns57fsX61, patient no. 16), exon 3 (c.631G $>$ T,
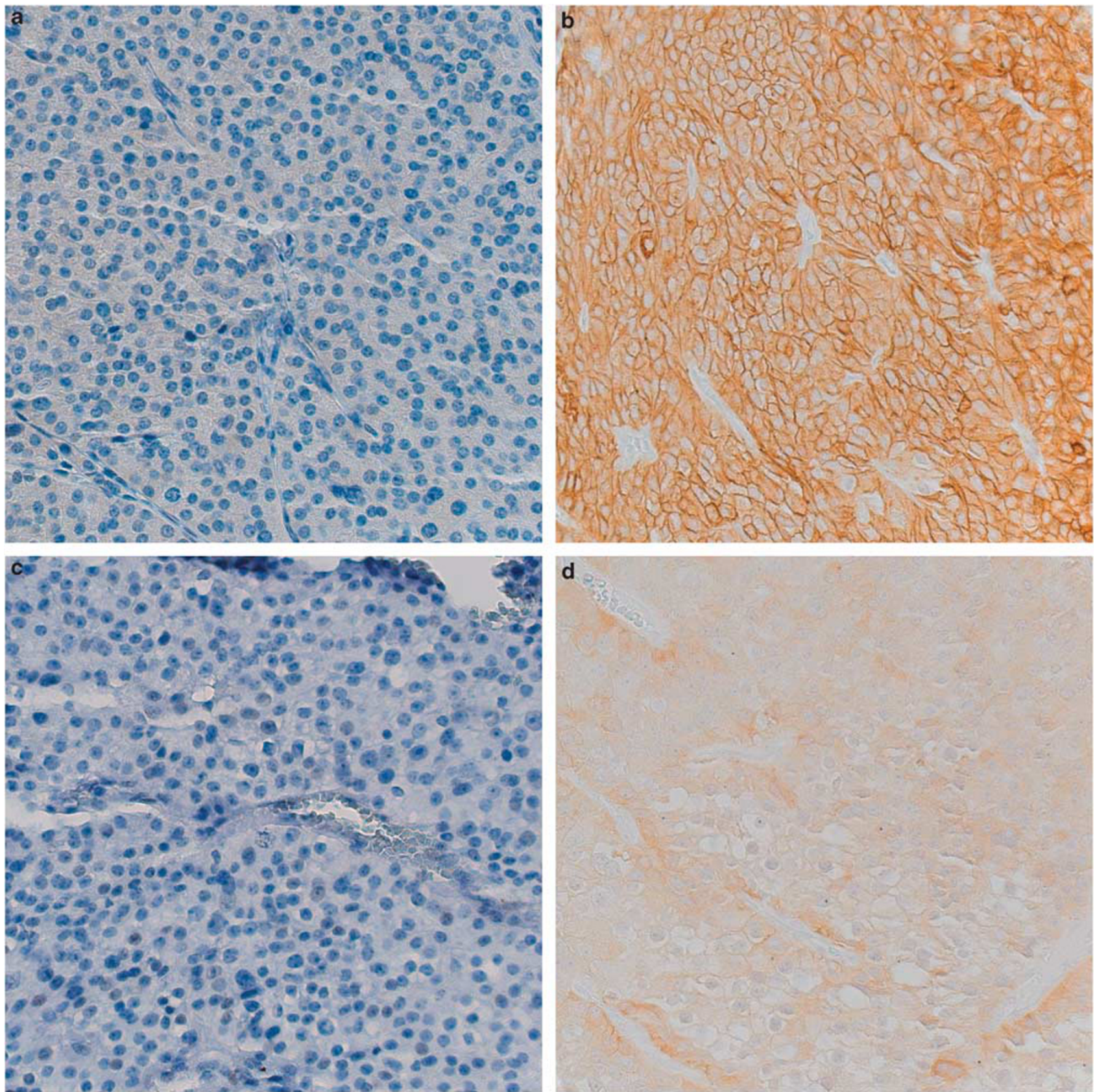

Figure 1 Staining with hematoxylin-eosin for CASR expression (a/c) and immunohistochemical (IHC) staining of CASR (b/d) in two patients with parathyroid carcinoma (patient $7(\mathbf{a} / \mathbf{b})$ and patient $1(\mathbf{c} / \mathbf{d})$ ) showing maintained membranous CASR expression in patient 7 $(\mathbf{a} / \mathbf{b})$ and loss of membranous CASR expression in patient $1(\mathbf{c} / \mathbf{d})$. Magnification $\times 25$. 

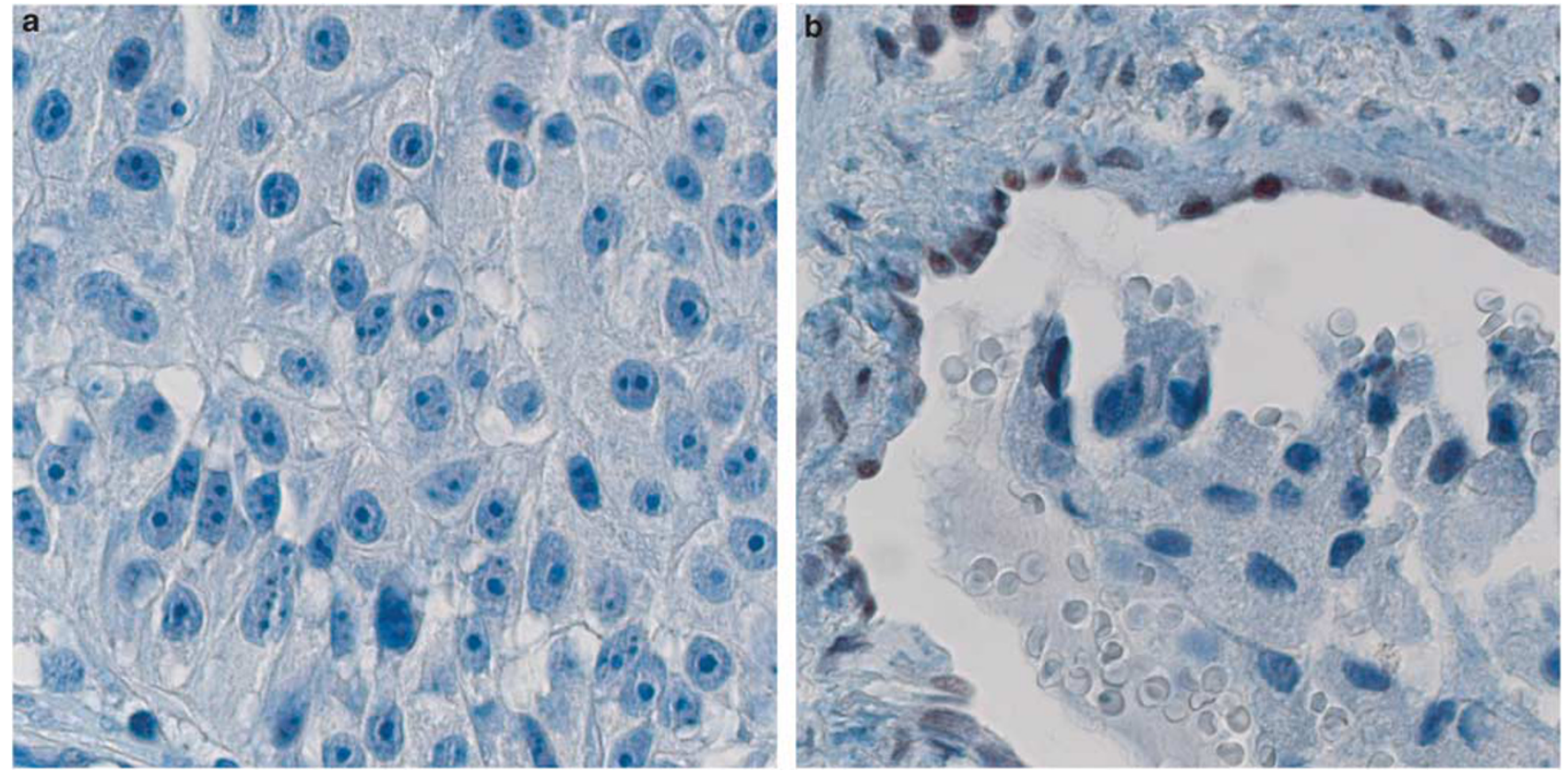

Figure 2 Immunohistochemical staining of parafibromin in a patient with parathyroid carcinoma (patient 23) showing global loss of parafibromin staining in the nuclei of parathyroid carcinoma cells (a) and in the nuclei of parathyroid carcinoma cells located intravascular (b). In contrast, the nuclei of the endothelial cells of the vascular wall stained positive for parafibromin (b). Magnification $\times 100$.

pVall211Phe, patient no. 5) and exon 9 (c.1256delG, pGly419fsX26, patient no. 14), respectively. ${ }^{14}$ The mutation specifics have been partly curated, that is, updated according to the latest published literature, in the Cosmic Database of the Sanger Institute (www.sanger.ac.uk/genetics/CGP/cosmic).

\section{Statistical Analysis}

Statistical analysis was performed using the SPSS 16 (SPSS, Chicago, IL, USA) software. Results are expressed as mean \pm s.d. unless otherwise stated. The $\chi^{2}$ test and Student's $t$-test were used for categorical variables and continuous variables. Disease-free survival (time to first local recurrence and/ or distant metastasis) and overall survival (time to death related to parathyroid carcinoma) was determined by the Kaplan-Meier method and $P$-values were calculated by the log-rank test. Cox regression analysis was used to determine hazard ratios (HRs).

\section{Results}

\section{Demographic Data}

A total of 12 women and 11 men, with a mean age of $51 \pm 16$ years at the time of diagnosis, were studied (Table 1). At initial presentation, polyuria and polydipsia were present in $50 \%$, tiredness in $31 \%$, muscle or bone pain in $25 \%$, constipation in $13 \%$ of patients and a neck swelling was palpable in 11 of 14 patients (79\%). Mean serum calcium concentration was $3.28 \pm 0.55 \mathrm{mmol} / \mathrm{l}$ (reference range
2.15-2.55 mmol/l), mean parathyroid hormone level was $75 \pm 57 \mathrm{pmol} / \mathrm{l}$ (reference range 1.5-8 pmol/l), mean alkaline phosphatase concentration was $386 \pm 297 \mathrm{U} / \mathrm{l}$ (reference range 40-120 U/1) and creatinine clearance was $<60 \mathrm{ml} / \mathrm{min}$ in $59 \%$ of patients.

\section{Surgical Data}

Of the 23 patients, 17 (74\%) had bilateral neck exploration and the remainder unilateral neck exploration. Parathyroidectomy was combined with hemithyroidectomy in 12 patients (52\%) because of thyroid invasion $(n=11)$ or preoperatively identified thyroid pathology $(n=1)$. Invasive growth of the tumor was observed intraoperatively in 14 of 21 patients $(67 \%)$, including invasion of the thyroid $(n=8)$, the recurrent laryngeal nerve $(n=2)$, the esophagus $(n=1)$, both the thyroid and esophagus $(n=1)$ and unspecified tissue $(n=2)$ (Table 1).

\section{Histopathological Data}

On pathological evaluation, cystic features were observed in 6 of the 23 tumors $(26 \%)$, and fibrotic bands were present in 19 of the 23 tumors (83\%). There was no mitotic activity in 7 of the 23 tumors $(30 \%)$, sporadic mitotic activity was observed in 10 $(44 \%)$ and $>1 / 10$ high-power field (HPF; more than 1 mitotic figure per HPF) was observed in 6 patients $(26 \%)$. Invasion of blood vessels was documented in 21 primary tumors (91\%). Four patients had local lymph node metastases. 
Table 1 Operative, pathological and follow-up data of patients with parathyroid carcinoma

\begin{tabular}{|c|c|c|c|c|c|c|c|c|c|c|c|}
\hline $\begin{array}{l}\text { Patient } \\
\text { number }\end{array}$ & $\begin{array}{l}\text { Age/ } \\
\text { sex }\end{array}$ & $\begin{array}{l}\text { Peroperative } \\
\text { invasive } \\
\text { growth }\end{array}$ & Vasoinvasion & $\begin{array}{l}\text { CASR } \\
\text { expression }\end{array}$ & $\begin{array}{l}\text { Parafibromin } \\
\text { loss }\end{array}$ & $\begin{array}{l}\text { Mutation } \\
\text { screening }\end{array}$ & $\begin{array}{l}\text { Development } \\
\text { of local } \\
\text { recurrence }\end{array}$ & $\begin{array}{l}\text { Development of } \\
\text { lymph node } \\
\text { metastasis }\end{array}$ & $\begin{array}{l}\text { Development of } \\
\text { distant } \\
\text { metastasis }\end{array}$ & $\begin{array}{l}\text { Follow- } \\
\text { up (years) }\end{array}$ & Outcome \\
\hline 1 & $30 / \mathrm{F}$ & Yes & Yes & Downregulation & Global & Germline HRPT2 & Yes & Yes & Yes & 5 & DOD \\
\hline 2 & $71 / \mathrm{F}$ & Yes & Yes & Normal & Focal & & Yes & No & No & 15 & DOD \\
\hline 3 & $74 / \mathrm{F}$ & Yes & Yes & Normal & Global & & No & No & Yes & 13 & $\mathrm{~A} / \mathrm{WD}$ \\
\hline 4 & $72 / \mathrm{F}$ & No & Yes & Normal & Focal & & No & No & No & 10 & $\mathrm{~A} / \mathrm{FOD}$ \\
\hline 5 & $77 / \mathrm{M}$ & Yes & Yes & Downregulation & Global & Somatic $M E N-1$ & No & Yes & Yes & 1 & DOD \\
\hline 6 & $29 / \mathrm{M}$ & Yes & Yes & Downregulation & Global & Somatic HRPT2 & Yes & Yes & Yes & 16 & $\mathrm{~A} / \mathrm{WD}$ \\
\hline 7 & $57 / \mathrm{M}$ & Yes & No & Normal & Global & & No & No & No & 13 & A/FOD \\
\hline 8 & $48 / \mathrm{M}$ & Yes & Yes & Normal & Focal & & No & No & No & 16 & $\mathrm{~A} / \mathrm{FOD}$ \\
\hline 9 & $32 / \mathrm{M}$ & No & Yes & Normal & Global & & Yes & No & No & 10 & $\mathrm{~A} / \mathrm{WD}$ \\
\hline 10 & $75 / \mathrm{M}$ & No & Yes & Normal & Focal & & No & No & No & 6.5 & D/other \\
\hline 11 & $66 / \mathrm{F}$ & No & Yes & Normal & Focal & & Yes & No & Yes & 8 & DOD \\
\hline 12 & $66 / \mathrm{F}$ & Yes & Yes & Normal & Global & & No & Yes & Yes & 13.5 & DOD \\
\hline 13 & $34 / \mathrm{M}$ & NA & Yes & Normal & Global & & Yes & Yes & Yes & 7 & DOD \\
\hline 14 & $50 / \mathrm{F}$ & Yes & Yes & Normal & Global & Somatic $M E N-1$ & No & No & Yes & 3.5 & DOD \\
\hline 15 & $29 / F$ & No & Yes & Downregulation & Global & & No & No & No & 1.5 & DOD \\
\hline 16 & $51 / \mathrm{F}$ & Yes & Yes & Normal & Focal & Somatic $M E N-1$ & No & No & No & 9 & $\mathrm{~A} / \mathrm{FOD}$ \\
\hline 17 & $54 / \mathrm{M}$ & No & Yes & Normal & Focal & & No & No & Yes & 13 & DOD \\
\hline 18 & $36 / \mathrm{F}$ & No & Yes & Normal & Global & & No & No & No & 18 & $\mathrm{~A} / \mathrm{FOD}$ \\
\hline 19 & $62 / \mathrm{F}$ & Yes & Yes & Downregulation & Global & & No & No & Yes & 1 & DOD \\
\hline 20 & $39 / \mathrm{M}$ & Yes & No & Normal & Focal & & No & No & No & 10 & $\mathrm{~A} / \mathrm{FOD}$ \\
\hline 21 & $41 / \mathrm{M}$ & Yes & Yes & Downregulation & Global & $\begin{array}{l}\text { Germline+somatic } \\
\text { HRPT2 }\end{array}$ & No & No & Yes & 3 & DOD \\
\hline 22 & $62 / \mathrm{M}$ & Yes & Yes & Normal & Focal & & No & No & No & 11 & A/FOD \\
\hline 23 & $36 / \mathrm{F}$ & NA & Yes & Downregulation & Global & Germline HRPT2 & Yes & No & No & 20 & D/other \\
\hline
\end{tabular}

NA: not available, ND: not determined, DOD: dead of disease, D/other: dead of other cause, A/WD: alive with disease, A/FOD: alive, free of disease 


\section{Disease-Free Survival and Survival}

Median duration of follow-up after initial parathyroidectomy was 10 years (range $1-20$ years). Of the 23 patients, 7 (30\%) developed local recurrence of the tumor after a median duration of 3 years (range 1-7 years) after initial parathyroidectomy. One or more distant metastasis developed in 11 patients $(48 \%)$ after a median duration of 2 years (range 0.5-13 years): lung metastasis $(n=10)$, lymph node metastasis $(n=5)$, bone metastasis $(n=2)$, liver metastasis $(n=1)$ and brain metastasis $(n=1)$. Disease-free survival was 96, 60 and $40 \%$, respectively, at 1, 5 and 10 years after initial parathyroidectomy.

Of the 23 patients, $13(57 \%)$ had died at the time of the study. In 11 of these patients (85\%), death was related to severe uncontrollable hypercalcemia. Death was unrelated to parathyroid carcinoma in the other two patients, who died respectively of septic shock and of a cerebrovascular accident, in the setting of normocalcemia. Overall survival rates were 91,74 and $65 \%$, respectively, at 1,5 and 10 years after initial parathyroidectomy.

There was no significant difference in survival and disease-free survival between patients who had a hemithyroidectomy and those who did not (61 vs $58 \%, P=0.7$ and 81 vs $67 \%, P=0.8$, respectively).

\section{Downregulation of CASR Expression}

Of the 23 patients, 7 (30\%) demonstrated downregulation of CASR expression in the resected primary tumor (Figure 3). Of these 7 patients, 5 also had somatic and/or germline HRPT2/CDC73 or MEN-1 mutations. Patients with downregulation of CASR expression demonstrated more global than focal parafibromin loss compared with patients with normal CASR expression (6/6 vs $7 / 16, P=0.02)$. At

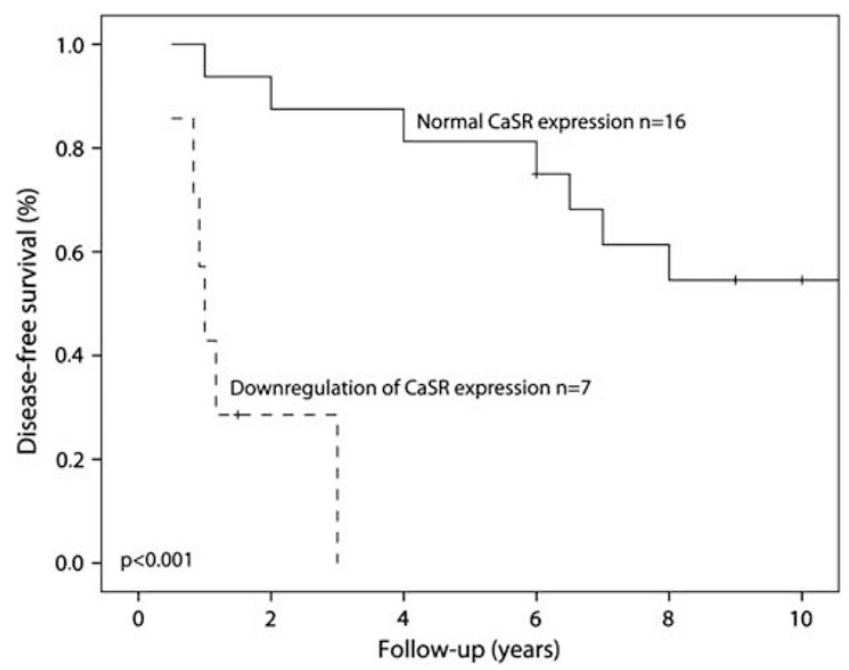

Figure 3 Significant decrease in 5-year disease-free survival in patients with downregulation of CASR expression compared with patients with normal CASR expression (81 vs $0 \%$ ). the time of initial surgery, positive lymph nodes were also more frequently found in patients with downregulation of CASR expression compared with patients with normal CASR expression (3/5 vs 1/16, $P<0.01$ ).

Of the 7 patients with downregulation of CASR expression, 6 developed local recurrence and/or distant metastasis compared with only 8 of the 16 patients with normal CASR expression (log-rank test, $P=0.1$ ). The time to development of a local recurrence and/or distant metastasis was significantly shorter in patients with downregulation of CASR expression compared with patients with normal CASR expression ( $1 \pm 1$ years VS $9 \pm 5$ years, $P<0.01)$. In addition, 5 of the 7 patients with downregulation of CASR expression and 6 of 16 patients with normal CASR expression had parathyroid carcinoma-related death at a mean of $2 \pm 2$ years and $10 \pm 5$ years, respectively, after initial surgery.

Both the 5-year disease-free survival and the 5 -year overall survival were lower in patients with downregulation of CASR expression compared with patients with normal CASR expression (81 vs 0\% and 94 vs 29\%, respectively, Figure 3). Downregulation of CASR expression was associated with a 16-fold increased risk of developing a local or distant metastasis (HR 16; 95\% CI 3-87; $P<0.01$ ) and a threefold increased risk of death (HR 2.9; $95 \%$ CI 0.8-9.7; $P=0.09$; Table 2).

\section{Loss of Parafibromin}

Parafibromin loss was determined in the resected primary tumor in all 23 patients. A total of 14 specimens showed global loss (Figure 2) and 9 showed focal loss of parafibromin staining. Of 14 patients with global parafibromin loss, 11 developed a local recurrence and/or distant metastasis compared with 3 of 9 patients with focal parafibromin loss (log-rank test $P=0.03$ ). Also, 8 of 14 patients with global parafibromin loss and 3 of 9 patients with focal parafibromin loss had a parathyroid carcinoma-related death (log-rank test $P=0.27$ ). Both the 5-year disease-free survival (89 vs 41\%) and the 5-year overall survival (100 vs 57\%) were lower in patients with global parafibromin loss compared with patients with focal parafibromin

Table 2 Association between prognostic factors and the development of local recurrence and/or distant metastasis

\begin{tabular}{lrc}
\hline & HR (95\% CI) & P-value \\
\hline Downregulation of CASR expression & $16.3(3.0-87.3)$ & $P=0.000$ \\
HRPT2/CDC73 mutation & $7.4(1.8-30.6)$ & $P=0.005$ \\
Global loss of parafibromin & $4.3(1.2-15.9)$ & $P=0.028$ \\
One or more of these prognostic & $4.3(1.2-15.9)$ & $P=0.028$
\end{tabular}

factors 


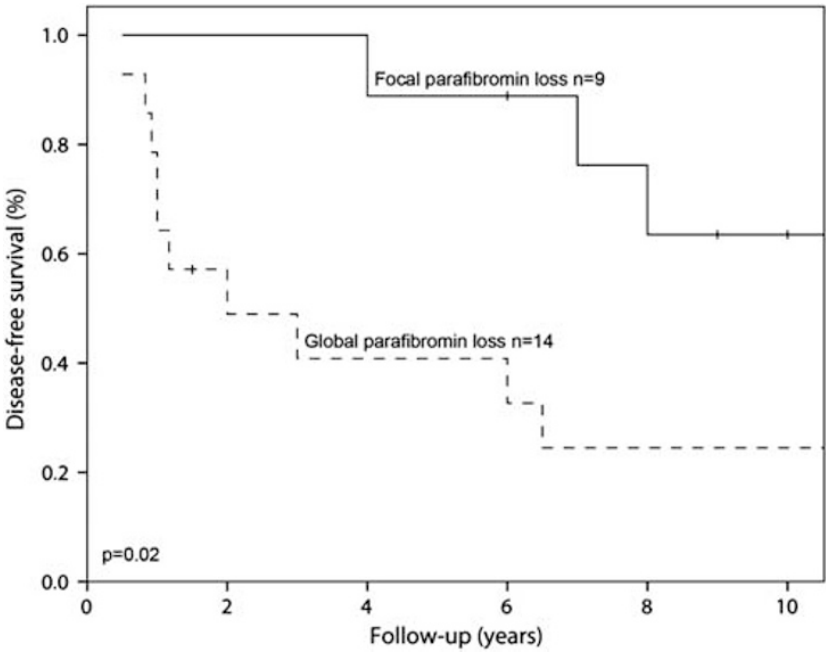

Figure 4 Significant decrease in 5-year disease-free survival in patients with global loss of parafibromin staining compared with patients with focal loss of this protein ( $89 \mathrm{vs} 40 \%$ ).

loss (Figure 4). Global parafibromin loss was associated with a fourfold increased risk of developing local recurrence and/or distant metastasis (HR 4.3; 95\% CI 1.2-15.9; $P=0.03$ ) and with a twofold increased risk of death (HR 2.0; 95\% CI 0.5-7.8; $P=0.3$; Table 2)

\section{HRPT2/CDC73 Mutation}

Of the 23 patients with parathyroid carcinoma, 2 had a germline HRPT2/CDC73 mutation, one had a somatic HRPT2/CDC73 mutation and one patient had both a germline and a somatic HRPT2/CDC73 mutation (Table 1). All four patients with the HRPT2/CDC73 mutation developed a local recurrence and/or distant metastases compared with only 10 of the 19 patients without this mutation (log-rank test: $P=0.08$ ). Time to development of a local recurrence and/or distant metastasis was shorter in patients with an HRPT2/CDC73 mutations compared with patients without this mutation $(1 \pm 1$ year vs $8 \pm 5$ years, $P<0.01$ ). Two of the 4 patients with an HRPT2/CDC73 mutation and 9 of 19 patients without this mutation had a parathyroid carcinoma-related death. Both the 5-year disease-free survival (73 vs $0 \%$ ) and the 5 -year overall survival (79 vs $50 \%$ ) were lower in patients with an HRPT2/CDC73 mutation compared with patients without this mutation (Figure 5). HRPT2/CDC73 mutations were associated with a sevenfold increased risk of developing local recurrence and/or distant metastasis (HR 7.4; 95\% CI 1.830.6; $P<0.01$; Table 2).

\section{MEN-1 Mutation}

Of the 23 patients with parathyroid carcinoma, 3 had a somatic $M E N-1$ mutation (Table 1). Patient 16

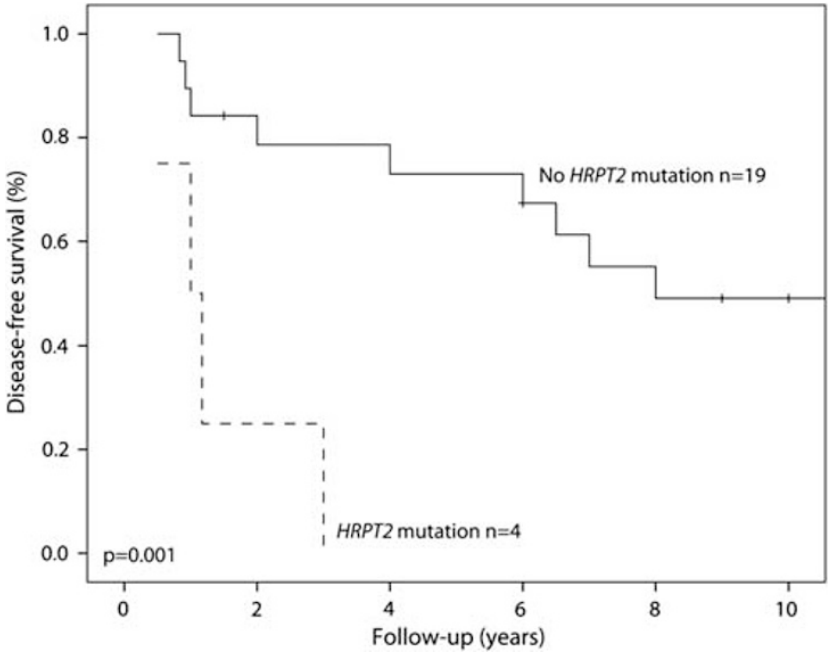

Figure 5 Significant decrease in 5-year disease-free survival in patients with germline and/or somatic HRPT2/CDC73 mutation compared with patients without a mutation in the HRPT2/CDC73 gene (73 vs $0 \%)$.

had intraoperative findings of invasion of the thyroid gland and pathological features of vasoinvasion, but did not develop local or distant metastases during 9 years of follow-up. Patient 14 had a palpable neck swelling at presentation, intraoperative findings of invasion, pathological features of vasoinvasion and developed lymph node, lung and bone metastases 11 months after initial parathyroidectomy. There was no response to chemotherapy and the patient died of uncontrollable hypercalcemia soon after the diagnosis of metastases. Patient 5 also had a palpable swelling in the neck, intraoperative findings of invasion, pathological features of vasoinvasion, a positive lymph node and developed lung and brain metastases 18 months after initial parathyroidectomy. The patient died of uncontrollable hypercalcemia 24 months later. In these three patients with somatic $M E N-1$ mutations, the 5-year disease-free survival and the 5-year overall survival were lower compared with patients without this mutation (respectively, 64 vs $33 \%$ and 80 vs 33\%). Somatic MEN-1 mutations were associated with a twofold increased risk of developing a local recurrence and/or distant metastasis (HR 1.7; 95\% CI 0.4-7.8; $P=0.5$ ) and a threefold increased risk of death (HR 3.3; 95\% CI 0.7-16.6; $P=0.15$; Table 2).

\section{Combined Effect of Negative Prognostic Factors}

Of 23 patients, 14 had one or more negative prognostic factors, but only 4 of the patients had a combination of an HRPT2/CDC73 mutation, global loss of parafibromin staining as well as downregulation of CASR expression. Both the 5-year disease-free survival and the 5-year overall survival were lower in patients with one or more negative 


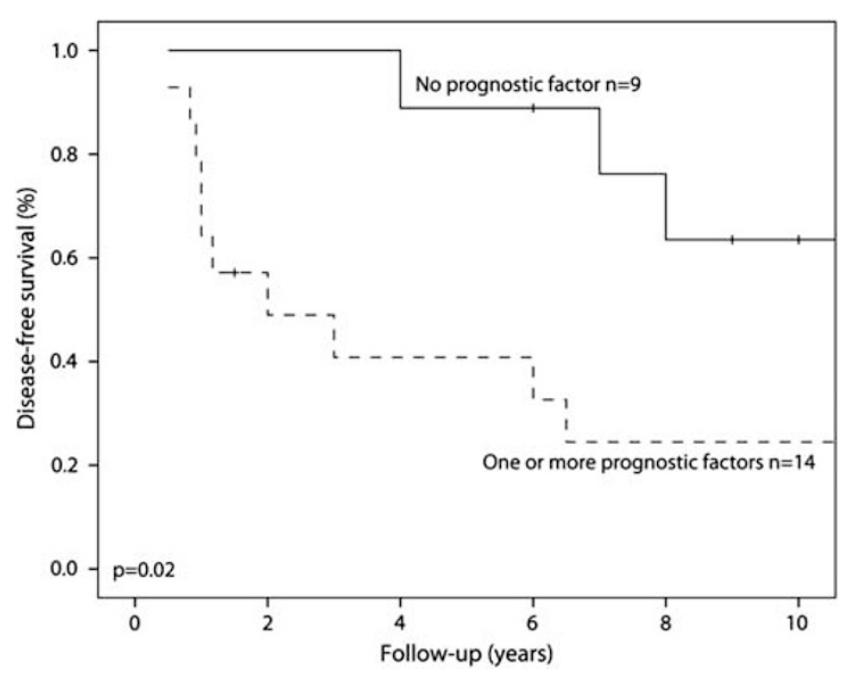

Figure 6 Significant decrease in 5-year disease-free survival in patients with one or more of the studied prognostic factors (germline and/or somatic HRPT2/CDC73 mutation, global loss of parafibromin staining or downregulation of CASR expression) compared with patients without one of these prognostic factors ( 89 vs $41 \%$ ).

prognostic factors compared with patients without one of these factors ( 89 vs $41 \%$ and 100 vs $57 \%$, respectively, Figure 6). Patients with one or more negative prognostic factors had a fourfold increased risk of developing local recurrence and/or distant metastasis (HR 4.3; 95\% CI 1.2-15.9; $P=0.03$; Table 2) and a twofold increased risk of death (HR $2.0 ; 95 \%$ CI $0.5-7.8 ; P=0.3$ ).

\section{Discussion}

Data from our study in patients with a WHO-based diagnosis of parathyroid carcinoma suggest that downregulation of CASR expression, global loss of parafibromin staining and the presence of germline and/or somatic HRPT2/CDC73 mutations are strong negative determinants of prognosis in patients with this malignancy, with clear associations with decreased disease-free survival and overall survival.

Although downregulation of CASR expression was present in only 7 of 23 patients, this molecular alteration was associated with a 16 -fold increased risk of developing local recurrence and/or distant metastasis. This suggests that this tumor marker represents a significant determinant of prognosis in parathyroid carcinoma. The CASR holds an important function in the control of calcium homeostasis by the parathyroid gland. Activation of the CASR by its binding with calcium ions leads to inhibition of parathyroid hormone secretion, parathyroid hormone gene expression and thus parathyroid hormone synthesis, and of parathyroid cell proliferation. ${ }^{23}$ Mutations in the CASR gene that result in a loss of function of the CASR, such as seen in familial benign hypercalcemia, increase the calcium-dependent set point for inhibition of parathyroid hormone release from the parathyroid cell. ${ }^{26}$ However, no mutations in the CASR gene have been so far identified in patients with parathyroid carcinomas. $^{27}$ It also remains unclear whether increased parathyroid cell proliferation leads to decreased CASR expression as observed in autonomous hyperparathyroidism in chronic renal failure, or whether decreased CASR expression leads to increased parathyroid cell proliferation and increased malignant potential in patients with parathyroid carcinoma. ${ }^{21,23}$ Notwithstanding, evaluating CASR expression may not only be used as a prognostic tool in the management of patients with parathyroid carcinoma, but may also serve the purpose of determining whether calcimimetics may have a role in the therapeutic management of these patients. In one patient in our series (patient no. 6) demonstrating loss of CASR expression in his primary tumor, the calcimimetic cinacalcet, prescribed at a maximum dose of $90 \mathrm{mg}$ twice daily, was indeed unable to decrease parathyroid hormone or calcium levels to any extent (personal observation).

HRPT2/CDC73 mutations are known to be associated with the HPT-JT syndrome. These mutations have also been found in $67-100 \%$ of patients with parathyroid carcinoma selected on the basis of local recurrence or distant metastasis. ${ }^{12,13,15}$ In contrast, in our study, in which patients were selected on the basis of the WHO histopathological criteria for parathyroid carcinoma, rather than on the basis of the presence of a local recurrence and/or of distant metastasis, we could only demonstrate a germline and/or somatic HRPT2/CDC73 mutation in 4 of the 23 patients (17\%). These were 4 of 14 patients who developed a local recurrence and/or distant metastasis during follow-up (29\%), suggesting that factors other than mutations in the HRPT2/CDC73 gene may also have a role in the tumorigenesis of parathyroid carcinoma. One of the limitations of our study is that we may not have identified all mutations in the HRPT2/CDC73 gene. The low mutation frequency in our population may thus be due to technical reasons, because of the nature of the paraffin-embedded tissue studied, leading to the inability to completely screen all DNA sequences of the HRPT2/CDC73 gene or screen the DNA for large somatic or germline genomic deletions. ${ }^{14}$ However, exons 1, 2 and 7, which harbor up to $85 \%$ of all somatic HRPT2/CDC73 mutations, ${ }^{12,15,18,28,29}$ were completely screened in all patients. Silencing of HRPT2/CDC73 because of promoter hypermethylation was also not studied, although this may not be relevant, as it was recently documented that hypermethylation does not have a role in loss of this gene. ${ }^{30}$ Although the numbers are small, our data do suggest that the presence of mutations in the HRPT2/CDC73 gene are associated with a significant decrease in disease-free survival and also survival, although the latter not significantly. These data suggest that mutations in this gene determine the 
malignant potential of a parathyroid carcinoma and may thus be used as a prognostic tool in this malignancy.

In patients with parathyroid carcinoma, most of the identified HRPT2/CDC73 mutations are predicted to prematurely truncate the parafibromin protein, ${ }^{12,13,16,28}$ which often lead to loss of parafibromin staining. Parafibromin is known to be involved in cell transcription, proliferation, differentiation and apoptosis, although the exact mechanism by which this protein increases the malignant potential of parathyroid carcinoma remains to be established. ${ }^{28}$ In our population, all patients with germline and/or somatic HRPT2/CDC73 mutations had global loss of parafibromin staining. We also found global loss of parafibromin staining in 10 patients in whom no mutations were identified, possibly pointing at the difficulties in capturing all mutations in our study population. In keeping with previous studies, which showed that loss of parafibromin staining was more frequently observed in patients who developed a local recurrence and/or distant metastasis compared with patients who did not $(62-96 \%$ vs $22-50 \%),{ }^{16,17,28}$ we indeed observed that patients with global loss of parafibromin staining had a short disease-free survival and survival. In contrast, focal loss of parafibromin staining did not appear to influence the clinical outcome. Moreover, in our experience, scoring of focal loss of parafibromin staining is difficult to use in daily practice, because of commonly encountered regional differences in immunohistochemical staining. In this case, it is the general staining pattern that is taken in consideration for decision making. Our data support the premise that focal loss of parafibromin staining might not be as useful as global loss of parafibromin staining as a diagnostic or prognostic tool in parathyroid carcinoma. Gill et $a l^{31}$ indeed proposed a scoring system in which focal loss would be considered positive, and concluded that complete absence of nuclear staining for parafibromin is diagnostic of parathyroid carcinoma or HPT-JTrelated (benign) tumor.

$M E N-1$ mutations are frequently found in familial $(95 \%)$ and also in sporadic adenomas $(20-30 \%)$, but so far only 4 cases of somatic $M E N-1$ mutations and 3 clinically suspected germline $M E N-1$ mutations have been reported in patients with parathyroid carcinoma. ${ }^{14,32-34}$ In our population, three patients demonstrated a somatic $M E N-1$ mutation. In all three patients, a diagnosis of parathyroid carcinoma was established on the basis of intraoperative findings of invasive growth $(100 \%)$, vasoinvasion $(100 \%)$ and distant metastasis $(67 \%)$. These findings suggest that patients with a somatic $M E N-1$ mutation are at risk of developing parathyroid carcinoma and that somatic $M E N-1$ mutations could have a role in parathyroid carcinogenesis.

Among many other markers, downregulation of CASR expression, global loss of parafibromin staining and mutation in the HRPT2/CDC73 gene are tools to assist in establishing a diagnosis of parathyroid carcinoma. Our data demonstrate a significant added value of these tumor markers as strong negative determinants of the malignant potential of parathyroid carcinomas. We therefore advocate the use of these prognostic tools in the management of patients with this malignancy. Findings from our study strengthen the link between molecular alterations and clinical course of patients with parathyroid carcinoma.

\section{Disclosure/conflict of interest}

The authors declare no conflict of interest.

\section{References}

1 Shane E. Clinical review 122: parathyroid carcinoma. J Clin Endocrinol Metab 2001;86:485-493.

2 Kebebew E. Parathyroid carcinoma. Curr Treat Options Oncol 2001;2:347-354.

3 Shane E, Bilezikian JP. Parathyroid carcinoma: a review of 62 patients. Endocr Rev 1982;3:218-226.

4 Schantz A, Castleman B. Parathyroid carcinoma. A study of 70 cases. Cancer 1973;31:600-605.

5 Wynne AG, van HJ, Carney JA, et al. Parathyroid carcinoma: clinical and pathologic features in 43 patients. Medicine (Baltimore) 1992;71:197-205.

6 Rawat N, Khetan N, Williams DW, et al. Parathyroid carcinoma. Br J Surg 2005;92:1345-1353.

7 Hundahl SA, Fleming ID, Fremgen AM, et al. Two hundred eighty-six cases of parathyroid carcinoma treated in the U.S. between 1985-1995 a National Cancer Data Base Report. The American College of Surgeons Commission on Cancer and the American Cancer Society. Cancer 1999;86:538-544.

8 Kebebew E, Arici C, Duh QY, et al. Localization and reoperation results for persistent and recurrent parathyroid carcinoma. Arch Surg 2001;136:878-885.

9 Chow E, Tsang RW, Brierley JD, et al. Parathyroid carcinoma-the Princess Margaret Hospital experience. Int J Radiat Oncol Biol Phys 1998;41:569-572.

10 August DA, Flynn SD, Jones MA, et al. Parathyroid carcinoma: the relationship of nuclear DNA content to clinical outcome. Surgery 1993;113:290-296.

11 DeLellis RA. World Health Organisation Classification of Tumours. Pathology and Genetics of Tumours of Endocrine Organs. AIRC Press: Lyon, 2006.

12 Shattuck TM, Valimaki S, Obara T, et al. Somatic and germ-line mutations of the HRPT2 gene in sporadic parathyroid carcinoma. N Engl J Med 2003;349: 1722-1729.

13 Howell VM, Haven CJ, Kahnoski K, et al. HRPT2 mutations are associated with malignancy in sporadic parathyroid tumours. J Med Genet 2003;40:657-663.

14 Haven CJ, van PM, Tan MH, et al. Identification of MEN1 and HRPT2 somatic mutations in paraffinembedded (sporadic) parathyroid carcinomas. Clin Endocrinol (Oxf) 2007;67:370-376.

15 Cetani F, Pardi E, Borsari S, et al. Genetic analyses of the HRPT2 gene in primary hyperparathyroidism: germline and somatic mutations in familial and sporadic parathyroid tumors. J Clin Endocrinol Metab 2004;89:5583-5591. 
16 Howell VM, Gill A, Clarkson A, et al. Accuracy of combined protein gene product 9.5 and parafibromin markers for immunohistochemical diagnosis of parathyroid carcinoma. J Clin Endocrinol Metab 2009;94:434-441.

17 Tan MH, Morrison C, Wang P, et al. Loss of parafibromin immunoreactivity is a distinguishing feature of parathyroid carcinoma. Clin Cancer Res 2004;10: 6629-6637.

18 Carpten JD, Robbins CM, Villablanca A, et al. HRPT2, encoding parafibromin, is mutated in hyperparathyroidism-jaw tumor syndrome. Nat Genet 2002;32: 676-680.

19 Iacobone M, Masi G, Barzon L, et al. Hyperparathyroidism-jaw tumor syndrome: a report of three large kindred. Langenbecks Arch Surg 2009;394:817-825.

20 Marx SJ, Simonds WF, Agarwal SK, et al. Hyperparathyroidism in hereditary syndromes: special expressions and special managements. J Bone Miner Res 2002;17(Suppl 2):N37-N43.

21 Haven CJ, van PM, Karperien M, et al. Differential expression of the calcium sensing receptor and combined loss of chromosomes $1 \mathrm{q}$ and $11 \mathrm{q}$ in parathyroid carcinoma. J Pathol 2004;202:86-94.

22 Haven CJ, Howell VM, Eilers PH, et al. Gene expression of parathyroid tumors: molecular subclassification and identification of the potential malignant phenotype. Cancer Res 2004;64:7405-7411.

23 Saidak Z, Mentaverri R, Brown EM. The role of the calcium-sensing receptor in the development and progression of cancer. Endocr Rev 2009;30:178-195.

24 Whitfield JF. Calcium, calcium-sensing receptor and colon cancer. Cancer Lett 2009;275:9-16.

25 Chakrabarty S, Radjendirane V, Appelman $\mathrm{H}$, et al. Extracellular calcium and calcium sensing receptor function in human colon carcinomas: promotion of E-cadherin expression and suppression of betacatenin/TCF activation. Cancer Res 2003;63:67-71.
26 Thakker RV. Genetics of endocrine and metabolic disorders: parathyroid. Rev Endocr Metab Disord 2004;5:37-51.

27 Hosokawa Y, Pollak MR, Brown EM, et al. Mutational analysis of the extracellular $\mathrm{Ca}(2+)$-sensing receptor gene in human parathyroid tumors. J Clin Endocrinol Metab 1995;80:3107-3110.

28 Newey PJ, Bowl MR, Cranston T, et al. Cell division cycle protein 73 homolog (CDC73) mutations in the hyperparathyroidism-jaw tumor syndrome (HPT-JT) and parathyroid tumors. Hum Mutat 2010;31:295-307.

29 Bradley KJ, Cavaco BM, Bowl MR, et al. Parafibromin mutations in hereditary hyperparathyroidism syndromes and parathyroid tumours. Clin Endocrinol (Oxf) 2006;64:299-306.

30 Hahn MA, Howell VM, Gill AJ, et al. CDC73/HRPT2 $\mathrm{CpG}$ island hypermethylation and mutation of $5^{\prime}$ untranslated sequence are uncommon mechanisms of silencing parafibromin in parathyroid tumors. Endocr Relat Cancer 2010;17:273-282.

31 Gill AJ, Clarkson A, Gimm O, et al. Loss of nuclear expression of parafibromin distinguishes parathyroid carcinomas and hyperparathyroidism-jaw tumor (HPT-JT) syndrome-related adenomas from sporadic parathyroid adenomas and hyperplasias. Am J Surg Pathol 2006;30:1140-1149.

32 Enomoto K, Uchino S, Ito A, et al. The surgical strategy and the molecular analysis of patients with parathyroid cancer. World J Surg 2010;34:2604-2610.

33 Dionisi S, Minisola S, Pepe J, et al. Concurrent parathyroid adenomas and carcinoma in the setting of multiple endocrine neoplasia type 1: presentation as hypercalcemic crisis. Mayo Clin Proc 2002;77: 866-869.

34 Agha A, Carpenter R, Bhattacharya S, et al. Parathyroid carcinoma in multiple endocrine neoplasia type 1 (MEN1) syndrome: two case reports of an unrecognised entity. J Endocrinol Invest 2007;30:145-149. 